\title{
Exogenous normal lymph alleviates lipopolysaccharide-induced acute lung injury through lessening the adhesion molecules ${ }^{1}$
}

\author{
Li-li Zhang', Zi-gang Zhao"I, Chun-yu Niu"II, Jing Zhang ${ }^{\mathrm{IV}}$
}

DOI: http://dx.doi.org/10.1590/S0102-86502014000500001

${ }^{I}$ Master, Lecturer, Institute of Microcirculation, Hebei North University, Zhangjiakou, China. Acquisition of data.

IIMaster, Full Professor, Institute of Microcirculation, Hebei North University, Zhangjiakou, China. Design of the study, manuscript writing, critical revision.

IIIPhD, Full Professor, Institute of Microcirculation, Hebei North University, Zhangjiakou, China. Design of the study, critical revision.

${ }^{\mathrm{IV}}$ Bachelor, Full Professor, Institute of Microcirculation, Hebei North University, Zhangjiakou, China. Design of the study, acquisition of data.

\begin{abstract}
PURPOSE: To evaluate the role of exogenous normal lymph (ENL) on lipopolysaccharide (LPS)-induced acute lung injury (ALI) in rats.

METHODS: ALI was induced by the jugular vein injection of LPS (iv, $15 \mathrm{mg} / \mathrm{kg})$ in rats of the LPS and LPS+ENL groups within $15 \mathrm{~min}$, then, ENL without cell components $(5 \mathrm{ml} / \mathrm{kg}$ ) was infused at the speed of $0.5 \mathrm{ml}$ per minute in the LPS+ENL group, the same amount of saline was administered in the LPS group. The rats in the sham group received the same surgical procedure and saline. The histomorphology and the levels of P-selectin, intercellular adhesion molecule-1 (ICAM-1), myeloperoxidase (MPO) in pulmonary tissue were assessed.

RESULTS: LPS induced pulmonary injury as well as increased the wet/dry weight ratio (W/D) and the levels of P-selectin, ICAM-1, and MPO in pulmonary tissues. These deleterious effects of LPS were significantly ameliorated by ENL treatment.

CONCLUSION: Exogenous normal lymph could markedly alleviate the acute lung injury induced by lipopolysaccharide, and its effects might be related to lessening the adhesion molecules.
\end{abstract}

Key words: Lymph. Lipopolysaccharides. Acute Lung Injury. P-Selectin. Intercellular Adhesion Molecule-1. Peroxidase. Rats. 


\section{Introduction}

Acute lung injury (ALI) often induced by trauma, hemorrhage, infection, inflammation, etc ${ }^{1}$, is major cause of acute respiratory failure, which increases the risk of morbidity and mortality in critically ill patients ${ }^{2}$. In generally, ALI is an excessive uncontrolled inflammatory response caused by several pro-inflammatory mediators ${ }^{3,4}$, however, the precise mechanisms of ALI remains poorly understood. Therefore, it is essential to explore the true mechanisms and effective treatment for $\mathrm{ALI}^{5}$. Our previous studies found that the exogenous normal lymph (ENL) from healthy dog has an alleviating effect on microcirculation disturbances in rats with disseminated intravascular coagulation (DIC), hemorrhagic shock and endotoxic shock ${ }^{6-8}$; in addition, the ENL could alleviate the acute kidney injury in rats with DIC and endotoxic shock ${ }^{9,10}$. However, whether the ENL could lessen the lipopolysaccharide (LPS)-induced ALI remains unclear. In consequence, the purpose of this study was to evaluate the effect of ENL on ALI induced by LPS in rats and reveal its mechanism.

\section{Methods}

All animal experiments were performed in accordance with the Animal Care Committee of Hebei North University, Zhangjiakou, China. All efforts were made to minimize suffering of animals.

Sixty healthy and specific pathogen free (SPF) male Wistar rats, $240 \mathrm{~g}$ to $300 \mathrm{~g}$, were purchased from the Chinese Academy of Medical Sciences Animal Breeding Center (Beijing, China), and maintained at an animal facility under barriersustained conditions with 12-h light/dark cycle at a standard conditions (temperature: $23 \pm 2{ }^{\circ} \mathrm{C}$, relative humidity: $40 \%-80 \%$ ), and free access to standard laboratory food and water. Before the animal experimentation, the rats were fasted for $12 \mathrm{~h}$, but were allowed free access to water. Besides, three healthy beagle dogs were used for the preparation of ENL.

\section{ENL preparation}

The healthy beagle dogs were anesthetized with pentobarbital sodium $(25 \mathrm{mg} / \mathrm{kg})$ intravenously after induction of anesthesia with ketamine. Then, a midline laparotomy was performed for normal mesenteric lymph collection continuously for $30 \mathrm{~min}$ as previously described ${ }^{6,8-10}$. Lymph samples were centrifuged for $15 \mathrm{~min}$ at $315 \mathrm{~g}$ to remove all cellular components and stored at $-80^{\circ} \mathrm{C}$ in refrigerator (Thermo Electron, MA, USA).

\section{ALI model establishment}

After anesthetization with pentobarbital sodium (1\%, 50 $\mathrm{mg} / \mathrm{kg}$ ), the rats were randomly divided into: sham group, LPS group, and LPS+ENL group $(n=20)$. The left jugular vein was aseptically separated from the surrounding tissues and cannulated using a microcatheter for LPS injection and ENL treatment. Meanwhile, the right carotid artery was also isolated and cannulated using a minimally heparinized polyethylene catheter for continuous monitoring of mean arterial pressure during the experiment. After a 30-min stabilization period, LPS $(15 \mathrm{mg} / \mathrm{kg}$, $10 \mathrm{mg} / \mathrm{ml}$ ) (Escherichia coli O111:B4) (Sigma, Milwaukee, WI, USA) was injected via the left jugular vein over 10 min using an infusion pump (ZCZ-50, Zhejiang University Medical Ltd., Hangzhou, China) in the LPS and LPS+ENL groups, then, $0.2 \mathrm{ml}$ of normal saline injected through the left jugular vein to Ensure that LPS was injected into the blood circulation. After $15 \mathrm{~min}$ of LPS injection, the ENL ( $5 \mathrm{ml} / \mathrm{kg}$ ) was infused via left jugular vein in the rats of the LPS+ENL group at the speed of $0.5 \mathrm{ml}$ per minute according with the previous experiments ${ }^{8,10}$; at the same time, the same amount of normal saline was infused to the LPS group rats. In the sham group, the rats received two injections of normal saline alone, instead of LPS or ENL.

\section{Pulmonary histomorphology evaluation}

At the $6 \mathrm{~h}$ after LPS injection or corresponding time points, the fixed position pulmonary tissue was obtained from rats and then fixed in 10\% neutral buffered formalin. After alcohol gradient dehydration and paraffin embedding, each paraffin block was processed into 5 - $\mu \mathrm{m}$-thick slices that were stained with hematoxylin and eosin (H\&E). The pulmonary morphological changes were observed with light microscopy (BH-2, Olympus, Tokyo, Japan) and pictures were taken using digital camera (4500, Nikon, Tokyo, Japan) from ten randomly chosen areas for per sample.

\section{Water content in pulmonary tissue determination}

At the $6 \mathrm{~h}$ after LPS injection or corresponding time points, the pulmonary tissue was harvested in a fixed position, and the wet weight was immediately measured using a precision electronic balance (LE225D, Sartorius Scientific LLC., Beijing, China) after blotting up it with filter paper. Then, the tissue was embedded in an electrical thermostatic drum wind drying oven (GZX-9070MBE, Boxun LTD., Shanghai, China), and was baked at $80^{\circ} \mathrm{C}$ for $12 \mathrm{~h}$ until the weight did not change. The dry weight of 
the same pulmonary tissue was measured again, and the wet/dry weight ratio (W/D) was calculated.

\section{Pulmonary homogenate preparation}

At $3 \mathrm{~h}$ or $6 \mathrm{~h}$ after LPS administration or corresponding time points, the pulmonary tissue was obtained in a fixed position, then, was homogenized in 1:9 (w/v) normal saline for 30s using a tissue homogenizer (FJ-200, Shanghai specimen model factory, Shanghai, China). The homogenate was centrifuged at $850 \mathrm{~g}$ at $0-4^{\circ} \mathrm{C}$ for $10 \mathrm{~min}$ using a supercentrifuge (Labofuge 400R, Thermo Fisher Scientific, San Jose, California, USA), the supernatant was frozen at $-80^{\circ} \mathrm{C}$ for further assays.

\section{P-selectin, ICAM-1 and MPO measurement}

The levels ofP-selectin(Yuanye Biotechnology, Shanghai, China) and intercellular adhesion molecule-1 (ICAM-1) (Bosde Biotechnology, Wuhan, China) in the pulmonary homogenate were determined with the enzyme-linked immunosorbent assay (ELISA) method according to the manufacturer's instructions. The MPO activity in the pulmonary homogenate was measured using the hydrogen peroxide method ${ }^{11}$ with the MPO kit (Jiancheng Biotechnology, Nanjing, China) according to the manufacturer's instructions. The protein content of homogenate was quantified with the Coomassie brilliant blue colorimetric method for the standardization of the above indices.

\section{Statistical analysis}

Data were reported as the Mean \pm standard deviation (SD) and were analyzed using SPSS version 16.0 software (Polar Engineering and Consulting Inc., Chicago, IL, USA). Intragroup comparisons were performed with one-way ANOVA. $P$ value of less than 0.05 was considered to be statistically significant.

\section{Results}

\section{Effect of ENL on pulmonary histomorphology in LPS rats}

The clear alveolar structures are showed in the sham group, and hemorrhage and effusion are not observed in alveolar spaces (Figure 1A). LPS caused several histopathological alterations characterized by alveolar wall thickening, inflammatory cells infiltration, and hemorrhage and effusion in alveolar spaces (Figure 1B). Similarly, treatment with ENL greatly improved these abnormal features of lung (Figure 1C).
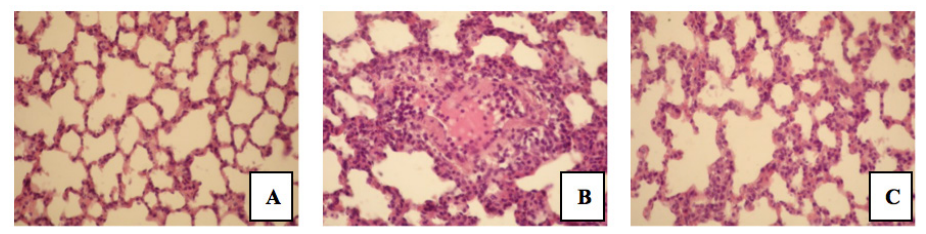

FIGURE 1 - Effect of exogenous normal lymph (ENL) on pulmonary histomorphology in rats (HE staining, $\times 400$ ). A: Sham group, alveolar structures show normal, and hemorrhage and effusion are not observed in alveolar spaces; B: Lipopolysaccharide (LPS) group, alveolar structures show disorder, alveolar septum shows thickening and inflammatory cells infiltration, and hemorrhage and effusion are appeared in alveolar spaces; C: LPS+ENL group, the changes of alveolar structures, hemorrhage and effusion are slighter than that of the LPS group.

\section{Effect of ENL on W/D of lung in LPS rats}

The W/D of lung in the LPS and LPS+ENL groups were obviously increased than that of the sham group $(P<0.05$, Figure 2 ), and the W/D of lung in the LPS+ENL group was significantly decreased compared with the LPS group $(P<0.05)$.

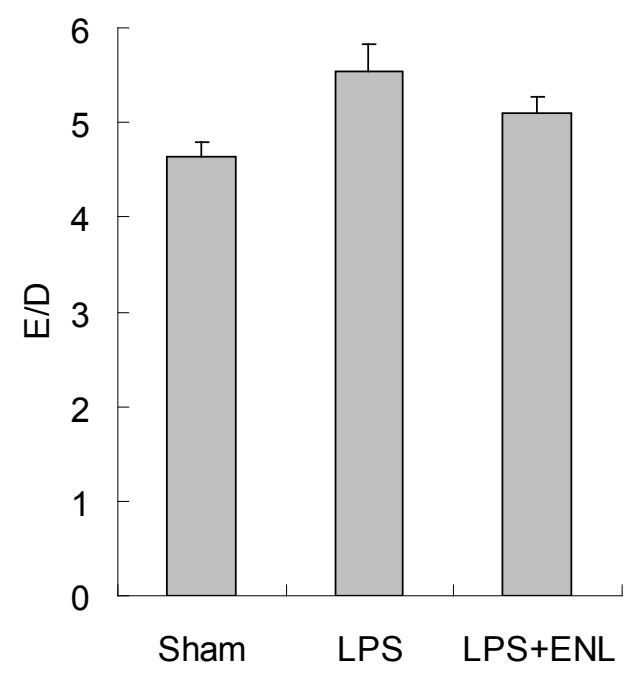

FIGURE 2 - Effect of exogenous normal lymph (ENL) on wet/dry weight ratio (W/D) of lung at $6 \mathrm{~h}$ after lipopolysaccharide (LPS) injection in rats. Mean $\pm \mathrm{SD}, n=10 .{ }^{*} P<0.05$ vs. the sham group; ${ }^{\#} P<0.05$ vs. LPS group.

\section{Effect of ENL on P-selection of lung in LPS rats}

At $3 \mathrm{~h}$ after LPS insults, the P-selection of hepatic homogenate in the LPS group was significantly higher than that of the sham group ( $P<0.03$, Figure 3$)$, and the P-selection of lung in the LPS+ENL group was significantly decreased compared with the LPS group $(P<0.05)$. 


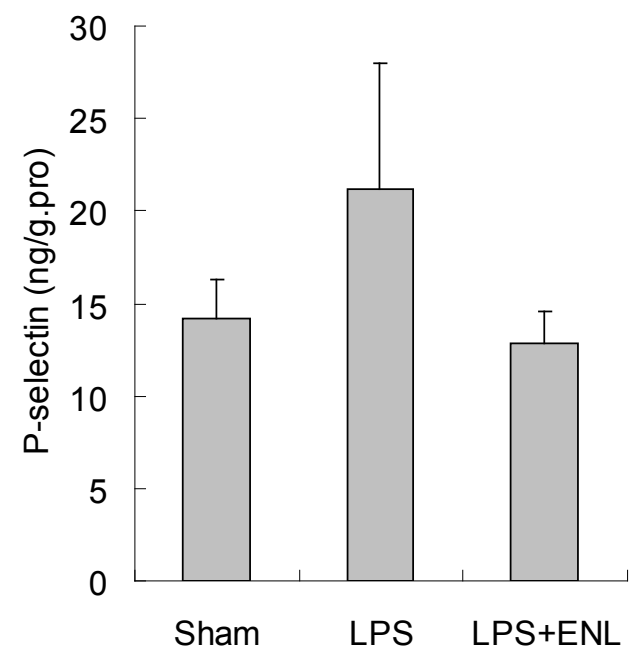

FIGURE 3 - Effect of exogenous normal lymph (ENL) on P-selection of pulmonary homogenate at $3 \mathrm{~h}$ after lipopolysaccharide (LPS) injection in rats. Mean $\pm \mathrm{SD}, n=10$. ${ }^{*} P<0.05 v s$. the sham group; ${ }^{\#} P<0.05 v$ s. the LPS group.

\section{Effect of ENL on ICAM-1 of lung in LPS rats}

At $3 \mathrm{~h}$ and $6 \mathrm{~h}$ after LPS administration, the ICAM-1 levels in pulmonary homogenate of the LPS group were significantly increased than the sham group ( $P<0.05$, Figure 4$)$, and the ICAM-1 levels in the LPS+ENL group were significantly lower compared with the LPS group $(P<0.05)$. Meanwhile, the ICAM-1 in the LPS+ENL group at $6 \mathrm{~h}$ was higher than that of the sham group $(P<0.05)$.

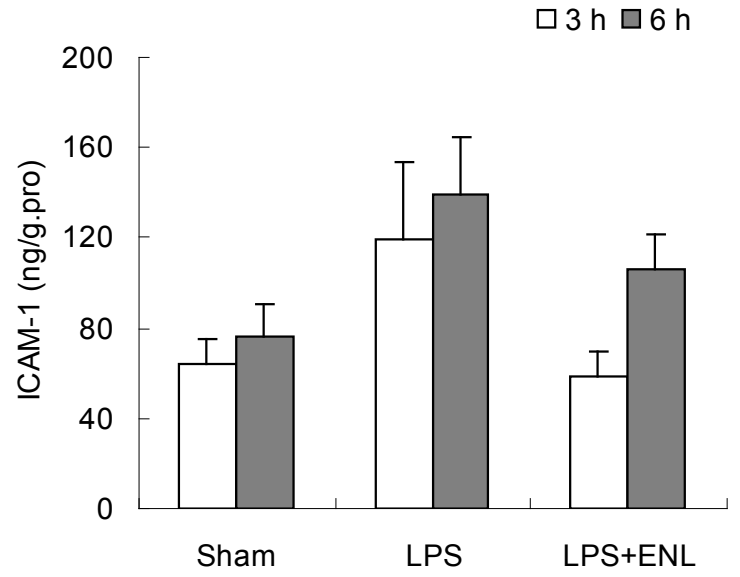

FIGURE 4 - Effect of exogenous normal lymph (ENL) on intercellular adhesion molecule-1 (ICAM-1) of pulmonary homogenate in rats. Mean \pm SD, $n=10$. ${ }^{*} P<0.05$ vs. the sham group; ${ }^{\#} P<0.05$ vs. the lipopolysaccharide (LPS) group.

\section{Effect of ENL on MPO of lung in LPS rats}

The Figure 5 as shown that the MPO activities of pulmonary homogenate in the LPS and LPS+ENL group at $3 \mathrm{~h}$ and $6 \mathrm{~h}$ after LPS administration were significantly increased than that of the sham group $(P<0.05)$; at the same time, the MPO activities of the LPS+ENL group at $3 \mathrm{~h}$ and $6 \mathrm{~h}$ were obviously lower compared with the LPS group $(P<0.05)$. $\square 3 \mathrm{~h} \square 6 \mathrm{~h}$

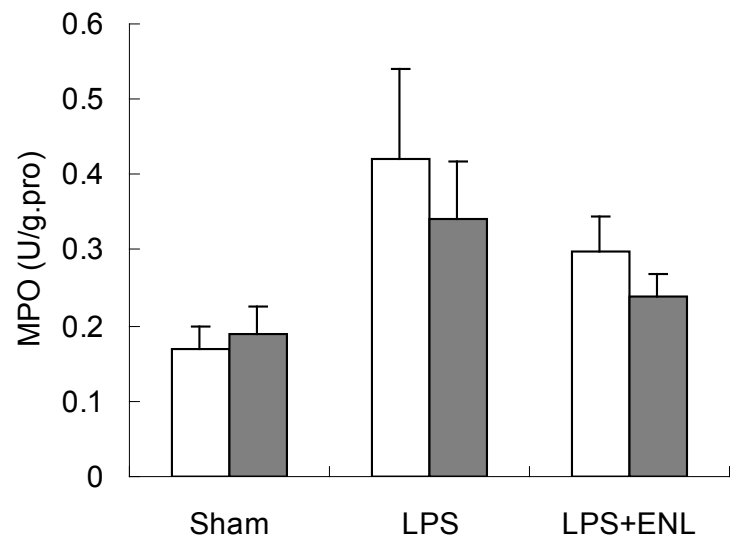

FIGURE 5 - Effect of exogenous normal lymph (ENL) on myeloperoxidase (MPO) activity of pulmonary homogenate in rats. Mean $\pm \mathrm{SD}, n=10$. * $P<0.05$ vs. the sham group; ${ }^{\#} P<0.05 v s$. the lipopolysaccharide (LPS) group.

\section{Discussion}

LPS, a cell wall component of gram-negative bacteria, is thought to play an important role in the development of $\mathrm{ALI}^{12-14}$. In current study, the administration of LPS via femoral vein, which reflecting enterogenous endotoxin translocation or exogenous toxins infection, caused several histopathological alterations characterized by alveolar wall thickening, inflammatory cells infiltration, and hemorrhage and effusion in alveolar spaces, these findings suggested the model was a useful and applicable experimental ALI model. Meanwhile, we found that the degree of pulmonary tissue damage was significantly attenuated by the administration of ENL, which suggested that the ALI induced by LPS-injection was prevented by treatment with ENL.

Furthermore, we found that the increased water content in lung induced by LPS administration was significantly attenuated by treatment with ENL. The result indicated that the mechanism of ALI was related to vascular endothelial cells (VECs) damage result in vascular hyper-permeability induced by $\operatorname{LPS}^{15,16}$, consequently, effusion in alveolar spaces was observed. Similarly, the treatment with ENL alleviated the VECs injury; therefore, the degree of effusion was decreased. However, the mechanism of ENL alleviating VECs injury is recommended for further study.

Previous studies show that LPS-induced ALI is considered a neutrophil-dependent ALI that contributes to local recruitment and activation of neutrophils ${ }^{17,18}$. Therefore, we explored the mechanisms by which ENL attenuates ALI from the neutrophil. After beening activated by LPS, VECs and neutrophils release cell-adhesion molecules such as P-selectin and ICAM-1, which promote the adhesion, aggregation, and sequestration of neutrophils in tissue ${ }^{19}$. In this study, there was increased P-selectin at $3 \mathrm{~h}$, as well as ICAM-1 levels and MPO activities at $3 \mathrm{~h}$ and 
$6 \mathrm{~h}$ in pulmonary tissue in the LPS group, while these increased indices in pulmonary tissue were reduced by ENL administration. In generally, MPO activity in tissue is significantly correlated with the number of neutrophil in affected tissues ${ }^{20}$. Therefore, these results suggested that LPS induced neutrophils adhesion via the increased P-selectin and ICAM-1 levels, at the same time, ENL administration is beneficial for reducing ALI by decreasing the neutrophil-mediated inflammatory response.

In addition, we found that ENL might reduce neutrophils attached to the venular wall and improve blood flow condition of microcirculation, and decrease the levels of P-selectin and ICAM1 in plasma $^{8}$, this result is an important supplement to the finding of this study.

\section{Conclusion}

An animal model of acute lung injury caused by the administration of lipopolysaccharide via femoral vein was established, and exogenous normal lymph could alleviate LPS- induced ALI through lessening the adhesion molecules levels and MPO activity.

\section{References}

1. Sotoudeh A, Takhtfooladi MA, Jahanshahi A, Asl AH, Takhtfooladi $\mathrm{HA}$, Khansari M. Effect of $\mathrm{N}$-acetylcysteine on lung injury induced by skeletal muscle ischemia- reperfusion. Histopathlogical study in rat model. Acta Cir Bras. 2012;27(2):168-71.

2. Rafat N, Tönshoff B, Bierhaus A, Beck GC. Endothelial progenitor cells in regeneration after acute lung injury: do they play a role? Am J Respir Cell Mol Biol. 2013;48(4):399-405.

3. Cabrera-Benitez NE, Pérez-Roth E, Casula M, Ramos-Nuez A, Ríos-Luci C, Rodríguez-Gallego C, Sologuren I, Jakubkiene V, Slutsky AS, Padrón JM, Villar J. Anti-inflammatory activity of a novel family of aryl ureas compounds in an endotoxin-induced airway epithelial cell injury model. PLoS One. 2012;7(11):e48468.

4. Tolle LB, Standiford TJ. Danger-associated molecular patterns (DAMPs) in acute lung injury. J Pathol. 2013;229(2):145-56.

5. von Dossow-Hanfstingl V. Advances in therapy for acute lung injury. Anesthesiol Clin. 2012;30(4):629-39.

6. Niu CY, Zhao ZG, Zhang YP, Hou YL, Li JJ, Jiang H, Zhang J. Exogenous normal lymph alleviates microcirculation disturbances and abnormal hemorheological properties in rats with disseminated intravascular coagulation. Braz J Med Biol Res. 2013;46(2):138-47.

7. Niu CY, Zhang J, Yao T, Fan G. The antagonistic effect of lymph on shock in rat. Lymphology. 1998;31(s):159-62.

8. Zhang LL, Zhao ZG, Niu CY, Zhang J. Interference effects of lymph plasma on endotoxic shock in rats. Zhongguo Ying Yong Sheng Li Xue Za Zhi. 2011;27(1):75-80.

9. Zhao ZG, Niu CY, Zhang YP, Hou YL, Li JJ, Jiang H. Exogenous normal lymph alleviating kidney injury by improving coagulation function in disseminated intravascular coagulation rats. Ren Fai. 2012;34(2):221-6

10. Zhao ZG, Niu CY, Zhang LL, Zhang J, Han R, Zhang YP, Hou YL. Exogenous normal lymph alleviates lipopolysaccharide-induced acute kidney injury in rats. Ren Fail. 2013;35(6):806-11.
11. Anderson BO, Brown JM, Shanley PF, Bensard DD, Harken AH. Marginating neutrophils are reversibly adherent to normal lung endothelium. Surgery. 1991;109(1):51-61.

12. Ma L, Wu XY, Zhang LH, Chen WM, Uchiyama A, Mashimo T, Fujino Y. Propofol exerts anti-inflammatory effects in rats with lipopolysaccharide-induced acute lung injury by inhibition of CD14 and TLR4 expression. Braz J Med Biol Res. 2013;46(3):299-305.

13. Li T, Zhang J, Feng J, Li Q, Wu L, Ye Q, Sun J, Lin Y, Zhang M, Huang R, Cheng J, Cao Y, Xiang G, Zhang J, Wu Q. Resveratrol reduces acute lung injury in a LPS-induced sepsis mouse model via activation of Sirt1. Mol Med Rep. 2013;7(6):1889-95.

14. Chen N, Wu Q, Chi G, Soromou LW, Hou J, Deng Y, Feng H. PrimeO-glucosylcimifugin attenuates lipopolysaccharide-induced acute lung injury in mice. Int Immunopharmacol. 2013;16(2):139-47.

15. Wang L, Taneja R, Wang W, Yao LJ, Veldhuizen RA, Gill SE, Fortin D, Inculet R, Malthaner R, Mehta S. Human alveolar epithelial cells attenuate pulmonary microvascular endothelial cell permeability under septic conditions. PLoS One. 2013;8(2):e55311.

16. Sun C, Beard RS Jr, McLean DL, Rigor RR, Konia T, Wu MH, Yuan SY. ADAM15 deficiency attenuates pulmonary hyperpermeability and acute lung injury in lipopolysaccharide-treated mice. Am J Physiol Lung Cell Mol Physiol. 2013;304(3):L135-42.

17. Abraham E. Neutrophils and acute lung injury. Crit Care Med. 2003;31(4 Suppl):195-9.

18. Grommes J, Soehnlein O. Contribution of neutrophils to acute lung injury. Mol Med. 2011;17(3-4):293-307.

19. Power C, Wang JH, Sookhai S, Wu QD, Redmond HP. Proinflammatory effects of bacterial lipoprotein on human neutrophil activation status, function and cytotoxic potential in vitro. Shock. 2001;15(6):461-6.

20. Reino DC, Pisarenko V, Palange D, Doucet D, Bonitz RP, Lu Q, Colorado I, Sheth SU, Chandler B, Kannan KB, Ramanathan M, Xu da Z, Deitch EA, Feinman R. Trauma hemorrhagic shock-induced lung injury involves a gut-lymph-induced TLR4 pathway in mice. PLoS One. 2011;6(8):e14829.

\section{Correspondence:}

Chunyu Niu

Institute of Microcirculation, Hebei North University

Zhangjiakou Hebei, 075000, China

Phone: (86)0313-4029168

ncylxf@126.com

lymphatics@126.com

Received: Dec 18, 2013

Review: Feb 20, 2014

Accepted: March 19, 2014

Conflict of interest: none

Financial sources: Scientific Research Projects of the Education Department in Hebei Province (2005311 and 2007407) and Foundation of Hundred Innovative Talents in Universities of Hebei Province (CPRC047 and CPRCII026)

${ }^{1}$ Research performed at Institute of Microcirculation, Hebei North University, Zhangjiakou Hebei, China. 